\title{
Profiles of Emotion Socialization Across Development and Longitudinal Associations with Youth Psychopathology
}

Laura G. McKee, $\mathrm{PhD}_{1}$, Karissa DiMarzio, $\mathrm{MS}_{2}$, Justin Parent, $\mathrm{PhD}_{2}$, Chelsea Dale, $\mathrm{MS}$ 2, Juliana Acosta, MS2, \& Jessica O’Leary, MA3

Submitted to Journal of Abnormal Child Psychology

1Department of Psychology, Georgia State University, Atlanta, GA

2Center for Children and Families, Florida International University, Miami, FL

3 Frances L. Hiatt School of Psychology, Clark University, Worcester, MA

\section{Declarations}

Funding: This research was supported by the Child and Adolescent Psychology Training and Research, Inc (CAPTR) and by NICHD F31HD082858 and F31HD101257.

Conflicts of Interest/Competing Interests: The authors declare that they have no conflicts of interest.

Availability of Data and Code: Anonymized data and code are available via Open Science Framework - https://osf.io/6f3c9/

Corresponding Author:

Name: Laura G. McKee, Ph.D.

Affiliation: Georgia State University

Email:1mckee1@gsu.edu 


\begin{abstract}
Although there is relative consensus in the literature regarding associations between certain emotion socialization (ES) strategies and youth behavioral health, there is very limited research from a person-centered perspective. To address this gap, the current study examined patterns of ES strategies in families and explored predictors and youth outcomes associated with those patterns. An economically-diverse sample of 229 predominately White mothers and fathers of youth aged 3-12 years was recruited online for a longitudinal study. Latent profile analysis was used to determine the optimal number of family clusters with similar ES profiles. Model fit supported a four-class model, which consisted of an Emotion Coaching profile, characterized by the lowest levels of putatively labeled unsupportive ES practices and the highest levels of putatively labeled supportive ES practices, a Moderate profile characterized by moderate levels of both unsupportive and supportive ES practices, a Limited Engagement profile characterized by low levels of both unsupportive and supportive ES practices, and an Emotion Dismissing profile characterized by the highest levels of unsupportive ES practices and the lowest levels of supportive ES practices. Cross-sectional and longitudinal differences were observed across the ES profiles with regard to demographic and parent emotional competence predictors and youth outcomes. The current study extends the literature on ES by providing evidence on how distinct ES profiles differentially predict youth behavioral health outcomes. Findings also underscore the importance of examining parent emotional competence as a catalyst for adaptive change in the family system.
\end{abstract}

Keywords: emotion socialization, youth psychopathology, latent profile analysis, emotion regulation, parenting 


\section{Profiles of Emotion Socialization Across Development and Longitudinal Associations with Youth Psychopathology}

The dynamic relationship between parents and their children is the primary training ground for youth as they develop emotional awareness and regulation skills. Work from numerous sub-disciplines, ranging from studies of attachment to vagal tone, has converged over the past half century to document how parent-child interactions unfold over time and are linked with youth emotion regulation (ER), and, in turn, adaptive functioning as well as psychopathology (Eisenberg et al., 2010). In more recent years, there has been a surge of interest specifically in emotion socialization (ES), a term encompassing how parents teach youth, directly and indirectly, to identify, regulate, and express emotions across contexts. In their influential heuristic model, Eisenberg, Cumberland, and Spinrad (1998) emphasized three emotion-related parenting practices that have been explored in numerous studies; namely, they identified ES as including the ways parents model emotional expressiveness, as well as how they discuss and react to the emotions expressed by their children.

In both Eisenberg et al.’s (1998) and Gottman et al.’s (1996) ES frameworks, youth ER, and in turn, indices of social-emotional competence are purportedly impacted by emotion-related parenting practices. In the literature that operationalizes ES as parental responses to youth emotion, specifically, some consensus has emerged regarding which strategies are considered supportive (e.g., problem-solving or encouraging reactions) versus unsupportive (e.g., punitive, neglectful, or minimizing reactions), with supportive or coaching strategies validating the child's emotional experience as well as providing guidance on how to deal with the emotion, and unsupportive or dismissive strategies potentially undermining the child's emotional experience by invalidating or punishing the expressed emotion (Eisenberg et al., 1998; Gottman et al., 
1996). In fact, there is increasing evidence demonstrating positive associations between supportive ES practices and youth adaptive regulation and behavioral health, and between unsupportive ES practices and maladaptive regulation and psychopathology (Eisenberg et al., 2010; Katz et al., 2012). For example, in an ethnically and economically diverse sample of families, Lunkenheimer et al. (2007) showed that when parents responded to youth negative emotion with invalidation, criticism, avoidance, or distraction, youth evidenced poorer ER skills as well as higher levels of internalizing and externalizing problems. In another study comparing ES practices in families with anxiety-disordered youth versus non-disordered youth, maternal emotion coaching (i.e., engaging with youth emotion with interest, support, and problem solving) was positively correlated with youth ER and negatively correlated with negativity and lability, while dismissive responses were negatively correlated with youth adaptive regulation and positively with negativity and lability. Furthermore, parents of anxiety-disordered youth were less likely to engage with the child's emotion in a supportive way and more likely to be dismissive, relative to parents of non-disordered youth (Hurrell et al., 2017). These studies represent only a sampling from a much larger literature suggesting that supportive ES strategies are associated with higher levels of emotional competence and lower levels of psychopathology (e.g., Cunningham et al., 2009; Fainsilber Katz et al., 2016), with the opposite being true for unsupportive strategies (e.g., Garside \& Klimes-Dougan, 2002; O’Neal \& Magai, 2005; O’Leary et al., 2019). Despite these noted trends, important contextual variables can influence the trajectories or outcomes associated with ES strategies, particularly those putatively labeled as unsupportive. For example, some evidence suggests that so-called unsupportive ES strategies can be innocuous or even adaptive depending on the context (e.g., Lugo-Candelas et al., 2015). 
Taken as a whole, ES serves as an important contributor to the development of children's emotional competence and related psychopathology. It is important to note, however, that the literature on ES has produced some mixed findings due to variability in ES responses across and within parents, and as a result of family demographics and contexts. For example, although supportive ES strategies are generally thought of as being beneficial to children, there is crosssectional and longitudinal support for a divergence model of ES in which low support from one parent may be mitigated by high support from the other, and may even optimize children's emotional understanding given their exposure to more diverse emotional expressions (McElwain, Halberstadt, \& Volling, 2007; Miller-Slough et al., 2017). Very little research, however, has examined the diversity of strategies each parent uses and/or the ways in which distinct withinparent ES strategies cluster or how they may interact to amplify or buffer the impact of other strategies. Data from a few notable exceptions (e.g., Garside \& Klimes-Dougan, 2002;

Lunkenheimer et al., 2007; O’Leary, 2020; O’Leary et al., 2019) do suggest that parents employ a variety of ES strategies. In one study, one-third of parents engaged in both supportive and unsupportive strategies with their children; additionally, coaching and dismissing responses interacted such that coaching, in the context of dismissing, was related to lower levels of youth internalizing symptoms and emotional lability/negativity (Lunkenheimer et al., 2007).

In two of the only three published works that take a person-centered approach, MillerSlough and colleagues showed that clusters typified by high levels of both supportive and unsupportive ES responses were predictive of positive change in youth effortful control (MillerSlough et al., 2015) and lower internalizing symptoms (Miller-Slough et al., 2017) relative to other patterns of ES responses. Consistent with suggestions that youth emotional competence is enhanced by access to both positive and low/moderate levels of negative emotional expression in 
the household (Morris et al., 2007), perhaps exposure to multiple, divergent socialization strategies increases youth's flexibility and facility with coping. This small, but significant, body of work suggests the importance of moving beyond a variable-centered approach to both (1) explore the ways parents combine strategies to socialize their children and (2) determine which patterns are linked with optimal or deleterious outcomes for youth. The current study builds from this base, taking a person-centered approach to identify profiles of ES strategies in families using latent profile analysis, and then using those profiles to predict youth outcomes. This method allows for consideration of the diversity of socialization messages children receive and how different combinations of responses are associated with youth ER and internalizing and externalizing symptoms. The study will also test predictors of group membership in the empirically-derived profiles. Two areas stand out as being ripe for study based on theoretic models and preliminary data (Eisenberg et al., 2008; Morris et al., 2007) - namely, family demographics (i.e., parent and youth sex; youth age; family income; parent education) and indices of parent emotional competence (i.e., ER; mindfulness).

With regard to demographics, a number of prior studies have suggested that mothers and fathers tend to use different socialization strategies, with fathers more likely, on average, to use more unsupportive strategies and mothers more likely to use supportive strategies (e.g., Baker et al., 2011; O'Leary et al., 2019). Child sex also appears to impact socialization messages (Cassano et al., 2007; O’Leary et al., 2019). Given that most studies, to date, have been crosssectional or have spanned only several years, the field lacks a guiding developmental model of ES (Katz et al., 2012). Nonetheless, comparisons across studies of children at different ages show that the same supportive strategies linked with adaptive outcomes for younger children may be less adaptive for older youth (e.g., Castro et al., 2018). Finally, regarding SES, several 
studies of ES suggest that low family income and less parent education are related to putatively labeled unsupportive reactions to youth negative emotion (Lugo-Candelas et al., 2015; Shaffer et al., 2012). Undoubtedly, emerging evidence suggests the importance of considering how a host of demographic variables relates to ES practices.

In addition to demographics, a burgeoning literature is demonstrating how parent emotional competence impacts ES practices. One small body of work examining parental psychopathology suggests that parents suffering from both Axis I and II disorders are more likely to use unsupportive strategies with their children (e.g., Breaux et al., 2016; Faro et al., 2019; Shaffer et al., 2012). Moving beyond associations between specific diagnoses and ES, it may prove beneficial to take a trans-diagnostic approach by examining, for instance, parent ER and mindfulness skills. Although these factors have been understudied as they relate to ES, a handful of parent training programs have augmented a traditional focus solely on behavioral principles to incorporate modules designed to enhance these parent resources (e.g., Coatsworth et al., 2015; Luby et al., 2018). The only two studies of which we are aware that used these indicators of parent emotional competence in relation to ES showed that emotion dysregulation is related to higher levels of unsupportive practices and lower levels of supportive practices (McKee et al., 2015), while mindful parenting is concurrently and longitudinally related to higher levels of supportive and lower levels of unsupportive ES responses (McKee et al., 2018). Additional data exploring predictors of ES behaviors are needed, as this information would enhance our understanding of what factors are most pertinent to parents' use of ES strategies and the ways in which these strategies influence youths' socioemotional functioning and later behavioral health.

In sum, although there is relative consensus in the literature regarding associations between certain ES responses and youth behavioral health, much of the work on ES to date has 
relied on aggregating data across parenting strategies and respondents, making it difficult to detect distinct patterns at the individual level. Furthermore, ES research has traditionally focused on processes in early or middle childhood, without attempting to distinguish whether ES responses are uniformly adaptive across ages or are sensitive to the changing demands of youth. To add to the extant literature and address the aforementioned limitations, the current study examined patterns of ES responses in families and explored predictors and youth outcomes associated with those patterns. Although we did not have specific hypotheses for the number of ES profiles we would observe, we did hypothesize that we would find patterns typified by (1) high levels of supportive and low levels of unsupportive ES similar to emotion coaching styles, (2) low levels of supportive and high levels of unsupportive responses similar to dismissive ES styles, and (3) high levels of both supportive and unsupportive responses, or a divergence pattern (Lunkenheimer et al., 2007; Miller-Slough et al., 2015, 2017). With regard to ES profile predictors, we hypothesized that mothers and parents with more emotional competence (as indicated by adaptive ER and higher dispositional mindfulness) would be associated with patterns of ES most similar to emotion coaching. Other demographic predictors were more exploratory in nature, given scant or discrepant past findings. Furthermore, based on the findings from variable-centered analyses, we expected that ES patterns most resembling emotion coaching would be predictive of higher levels of youth adaptive ER and lower levels of internalizing and externalizing symptoms and negativity/lability.

\section{Method}

\section{Participants}

A sample of 229 parents $\left(\mathrm{M}_{\text {parentage }}=34.14, \mathrm{SD}=6.42\right.$, range $=20-56 ; 37.1 \%$ fathers $)$ of youth aged 3 to 12 (Mchildage $=7.15 ; \mathrm{SD}=2.83 ; 51.1 \%$ female) from a larger study on the 
assessment of parenting was recruited through Amazon's Mechanical Turk (MTurk). With regard to race/ethnicity, $83.8 \%$ of parents identified as White, $7.0 \%$ as Black, $0.4 \%$ American Indian/Alaskan Native, 3.1\% Asian, and 5.7\% Hispanic. Approximately $60 \%$ of parents had at least a college education, $80.8 \%$ of families endorsed at least one co-parent, and $85.5 \%$ reported cohabitating with a partner or being married. Further, $22.7 \%$ reported currently being unemployed, $49.4 \%$ reported an annual income of at least $\$ 50,000$, with an average of 1.79 ( $\mathrm{SD}=.877$; range of 1- 5) children per family (see Supplemental Table S1 for complete details).

\section{Procedure}

Parents were recruited in 2015 via MTurk, a crowdsourcing application in the social sciences that has been demonstrated to be as reliable as traditional methods of data collection (Buhrmester et al., 2011), including with data on youth psychopathology (Parent et al., 2017). To participate, parents had to be a resident of the United States and have a task approval rating of $95 \%$ or higher on MTurk. All participants received $\$ 4.00$ and $\$ 8.00$ as compensation for their time and effort upon completion of the 8- and 12-month surveys, respectively. One child was randomly selected by a computer algorithm for parents who reported having multiple children in the desired age range; parents based survey responses on the selected child across all waves of the study. Ten attention check items were included in each survey. Data from participants were excluded as a result of inaccurate reporting on these items. Additional participants were excluded for reporting different demographic characteristics in two or more of the administered surveys (sample statistics are reported for families that passed these quality checks).

\section{Measures}

Demographic Information. Parents reported on their own demographics as well as those of their child (e.g., sex, age) and overall family (e.g., household income, family size). 
Emotion Socialization Strategies. The Coping with Children's Negative Emotions Scale

(CCNES; Fabes et al., 1990) is a self-report measure that gauges how parents respond to children's negative emotions in distressing situations. Parents respond to 12 hypothetical parentchild scenarios by indicating how likely, from 1 "very unlikely" to 7 "very likely", they would be to engage in 6 different response options (representing the 6 subscales) in each situation (e.g., their child is angry that he/she could not attend a friend's birthday party due to being sick or hurt). The CCNES is comprised of six subscales assessing different parental reactions: 1) emotion-focused reactions (e.g., "I would soothe my child and do something fun with him/her to make him/her feel better about missing the party"), 2) problem-focused reactions (e.g., "I would help my child think about ways that he/she can still be with friends"), 3) expressive encouragement (e.g., "I would encourage my child to express his/her feelings of anger and frustration"), (4) distress reactions (e.g., "I would get angry at my child"), (5) punitive reactions (e.g., "I would send my child to his/her room to cool off"), and (6) minimization reactions (e.g., "I would tell my child not to make a big deal out of missing the party"). Mean scores for each subscale range from 1-7, with higher scores indicative of the reaction being more normative of the parent. In addition, responses are often grouped into the two broader domains of supportive (i.e., emotion- and problem-focused reactions, expressive encouragement) and unsupportive ES practices (i.e., minimization, distress and punitive reactions) (Leerkes et al., 2014). The CCNES has demonstrated good internal reliability and has been shown to be sensitive to change over time (e.g., Denham \& Kochanoff, 2002; Herbert et al., 2013). The omega coefficients for the current study were .95 for supportive ES practices and .90 for unsupportive practices.

Parent Emotion Regulation. The Brief Difficulties with Emotion Regulation Scale (DERS-18; Victor \& Klonsky, 2016) is an 18-item measure that assesses parents' abilities to 
identify, express and regulate their emotions. Parents provide responses using a 5-point Likert scale $(1=$ almost never, 5 = almost always $)$ indicating how often each item applies to them (e.g., "When I'm upset, I have difficulty focusing on other things"). Higher scores indicate poorer ER. The DERS-18 has previously demonstrated excellent internal reliability and validity (Victor \& Klonsky, 2016). The omega coefficient for the current study was .93.

Parent Mindfulness. The Mindfulness Attention and Awareness Scale (MAAS; Brown \& Ryan, 2003) is a 15-item assessment of parents' dispositional mindfulness. Parents provide responses using a 6-point Likert scale $(1=$ almost always, $6=$ almost never $)$, indicating how frequently they experienced each statement (e.g., "I find it difficult to stay focused on what's happening in the present"). Higher scores reflect higher levels of mindfulness. The MAAS has demonstrated good internal consistency $(\alpha=.80-.90)$ as well as convergent and discriminant validity (Brown \& Ryan, 2003). The omega coefficient for the current study was .93.

Youth Emotion Regulation. The Emotion Regulation Checklist (ERC; Shields \& Cicchetti, 1997) is a 24-item assessment of the intensity, lability, flexibility, and appropriateness of a child's emotions. Parents indicate how often their child exhibits each behavior (e.g., "can recover quickly from episodes of upset or distress") using a 4-point Likert scale (1 = never, $4=$ almost always). Two subscales, negativity/lability (e.g., "Is prone to angry outbursts/tantrums easily") and ER (e.g., "Can say when s/he is feeling sad, angry or mad, fearful or afraid") are calculated with sum scores; higher values indicate higher negativity and competence, respectively. The ERC has strong psychometric properties (Shields \& Cicchetti, 1997). The omega coefficients for the ER and negativity/lability subscales were .77 and .87 , respectively.

Youth Psychopathology. Indicators of youth internalizing and externalizing problems were drawn from the Brief Problem Monitor (BPM; Achenbach et al., 2011). Parents respond to 
19 questions by evaluating internalizing, externalizing, attention and overall total problems. Items are rated on a 3 -point scale $(0=$ not true, $1=$ somewhat true, or 2 -very true $)$. Research supports the reliability and validity of test scores, including large correlations with corresponding longer scales (e.g., Child Behavior Checklist) as well as with diagnoses obtained from a structured diagnostic interview (Achenbach et al., 2011; Chorpita et al., 2010). Internal consistency for subscales at the 8- and 12-month follow-ups ranged from .82 to .88 .

\section{Data Analytic Plan}

The current study employed latent profile analyses (LPA) to identify constellations or profiles of ES strategies and their associations with covariates or predictors and with distal child psychopathology outcomes. LPA analyses allow variables that have similar indicator means and variances to cluster so that group patterns can be more easily identified.

Profile Enumeration. To determine the optimal number of profiles, we utilized the LoMendel-Ruben adjusted likelihood ratio test (LMR-A; Lo, Mendell, \& Rubin, 2001), the bootstrap likelihood ratio test (BLRT), the Bayesian Information Criterion (BIC), the Akaike Information Criteria (AIC), and entropy to select the best fitting model (see Table 1). Specifically, the LMR-A indicates statistically significant improvements $(p$-value $<.05)$ in a model in comparison to the model with one fewer profile. Similarly, a statistically significant BLRT indicates superiority in a model when compared to the model with one fewer profile. The AIC and BIC aid in determining model fit, with lower values on each index indicating better relative fit. Further, entropy determines the accuracy of classifying individuals into the profiles identified in each model, with values closer to 1 indicating more certainty in group division.

Profile Predictors and Outcomes. For the current study, we employed Vermunt's threestep approach (Vermunt, 2010) in Mplus for examining predictors. Specifically, once profiles 
were determined, cases were assigned to these profiles based on posterior probabilities. Following that, parent and youth covariates were introduced as predictors of the categorical latent class variable without needing to hard-classify nor resulting in distortion of profiles.

Regarding prediction of distal child outcomes, we used a three-step approach (Bakk et al., 2013) to examine the cross-sectional and longitudinal impact of latent parenting profiles on youth psychopathology and ER. Specifically, youth psychopathology and ER at waves three and four, and the stability of youth psychopathology and ER across time were included at the latent class level so that estimates were unbiased by classification inaccuracy and without distorting class solution. Two separate models were tested - first with child internalizing and externalizing problems and second with child emotional negativity and ER. Each model held the covariances between outcomes to be equal across profiles. Finally, child age was introduced as a moderator of the association between ES profile and child psychopathology and ER outcomes.

\section{Results}

\section{Latent Profiles}

Latent profile analyses (LPA) were conducted using Mplus version 8.3

(Muthén \& Muthén, 2017) to determine the optimal number of clusters of families with similar ES profiles. ES subscale scores were entered into the LPA models, which ranged from one to seven profiles and were run with a minimum of 200 random starts. Fit indices for the profiles are presented in Table 1. All models demonstrated acceptable entropy. Additionally, AIC and BLRT continued to support models with more classes. The BIC supported the four, five, and six class models as having a similar fit, with the five-class model the best fitting. However, upon examination of the 4 through 7 class models, it was clear that there was a limited separation between several classes in the more complex models (see Supplemental Figures S1-3 for 
complete details). Further, after the introduction of profile covariates, described in more detail below, the five and six-class models shifted considerably to have classes with fewer than $5 \%$ of the sample. Finally, based on fit, ease of interpretation, and robustness to covariates, we decided that the four-class model was the most theoretically and empirically defensible (see Figure 1).

An Emotion Coaching profile (28\%) was defined by the lowest levels of unsupportive ES and the highest levels of supportive ES. An Emotion Dismissing profile (16\%) had the highest levels of unsupportive and the lowest levels of supportive ES practices. A Moderate profile (31\%) exhibited moderate levels of both supportive and unsupportive ES with relatively low levels of emotion encouragement but relatively high levels of problem-focused reactions. A Limited Engagement profile (25\%) demonstrated low levels of both supportive and unsupportive ES. In other words, the Limited profile reported similar levels of unsupportive ES as the Emotion Coaching profile, but this was paired with levels of supportive ES that were similar to the Emotion Dismissing profile (save for clear separation for problem-focused reactions).

\section{Profile Predictors}

Following profile enumeration, we explored family demographic (i.e., parent and child sex, child age, parent education, family income) and parent emotional competence (i.e., mindfulness and ER) indicators as predictors of ES profile membership using multinomial logistic regression via Vermunt's three-step approach (see Table 2 for complete results).

Regarding family demographic predictors, parent sex was a statistically significant predictor of ES profile membership. Specifically, although mothers and fathers had similar probabilities of being in the Limited profile (24\% and $27 \%$, respectively), fathers had a higher likelihood of being in the Dismissing profile (26\% compared to $10 \%)$. Mothers had a higher probability of being in the Moderate (34\% compared to $25 \%$ ) and Coaching (31\% compared to 
$22 \%$ ) profiles. Additionally, child age was associated with profile membership: the probability of being in the Dismissing profile increased with child age (e.g., $10 \%$ for 4 -year-olds, $15 \%$ for 7 year-olds, and $22 \%$ for 10 -year-olds). The likelihood of being in the Coaching profile decreased with child age (e.g., $35 \%$ for age $4,28 \%$ for 7 , and $21 \%$ for 10 ). We also explored child sex as a predictor and found that parents of boys and girls were equally represented across the profiles.

Regarding family SES, family poverty status (i.e., being within $150 \%$ of the federal poverty line based on income and family size $=1$, above $150 \%=0$ ) was a statistically significant predictor of ES parenting profile membership. Specifically, higher-income families had a lower likelihood of being in the Dismissing profile (23\% compared to $13 \%$ ) as well as an increased probability of being in the Limited profile (30\% compared to $15 \%)$. Further, low-income families were more likely to be in the Coaching profile (35\% compared to $25 \%$ ) and a similar likelihood of being in the Moderate profile as higher-income families (27\% compared to $32 \%)$. Parent education, an additional indicator of family SES, was also associated with ES profile such that parents with a college education were more likely to be in the Moderate profile (34\% compared to $26 \%$ ) and less likely to be in the Coaching profile ( $22 \%$ compared to $37 \%$ ).

Finally, concerning parental emotional competence, the probability of being in the Emotion Coaching profile dramatically increased with higher levels of parental dispositional mindfulness (see Figure 2a). Specifically, parents with low levels of mindfulness had a 7\% probability of being in the Emotion Coaching profile, whereas parents with high levels had a $51 \%$ probability. The increase in the probability of being in the Emotion Coaching profile as parental mindfulness increases was paired with a decrease in the probability of being in the Moderate profile. In contrast, with respect to parent ER, the probability of being in the Emotion Dismissing profile substantially increased for parents with high levels of emotional dysregulation 
(see Figure 2b). For example, the likelihood of a parent being in the Dismissing profile was 3\% for low, 11\% for average, and 30\% for high emotion dysregulation (+1SD above the mean).

\section{Distal Child Psychopathology Outcomes}

Longitudinal associations between ES profile and child outcomes (e.g., internalizing, externalizing, ER and negativity/lability) were explored. Table 3 includes the Wald's chi-square tests of parameter equality results for all outcomes. Significant Wald's chi-square tests represent a difference in a specific parameter across profiles. Table 4 includes complete model results for paths estimated within each class. Overall, ES profile was associated with child emotional negativity, and both child internalizing and externalizing problems. The Emotion Dismissing profile was associated with the highest levels of child psychopathology and emotional negativity, while the Emotion Coaching profile was associated with the lowest levels. The Moderate and Limited profiles evidenced similar child outcomes, with child psychopathology levels falling inbetween the Emotion Coaching and Emotion Dismissing profiles. Cross-sectional associations showed similar patterns of results and included child ER as an additional significant outcome. Figure 3 depicts mean Z-scores for child outcomes for each profile.

Developmental moderation. Lastly, we explored if youth age moderated the association between ES profile and youth outcomes. For this analysis, we hard-classified families based on their most likely profile and then tested linear regression moderation models in R using Jamovi. For these models, wave 3 of each outcome served as the covariate, and the interaction between profile and child age was of primary interest. The associations between ES profile and child internalizing and externalizing problems were not moderated by child age, as evidenced by equivalent results across developmental stages (see Supplemental Tables S2-4). However, the association between ES practices profile and youth emotional negativity was moderated by child 
age (see Table 5). The form of the interaction was such that the Dismissing profile had an increasingly detrimental impact on youth emotional negativity as children transition to middle childhood and early adolescence (see Supplemental Figure S5 for details).

\section{Discussion}

The current study utilized a person-centered approach to examine patterns of ES in families and explore predictors and youth outcomes associated with those patterns. Although we did not have specific hypotheses related to the number of ES profiles that would be observed, we did expect to see patterns roughly consistent with emotion coaching, emotion dismissing, and a divergence model. These expectations were partially supported with findings revealing four distinct ES profiles: an Emotion Coaching profile characterized by parents who exhibited the lowest levels of unsupportive ES and the highest levels of supportive ES; a Moderate profile characterized by parents who exhibited moderate levels of both supportive and unsupportive ES practices; a Limited Engagement profile characterized by parents who exhibited low levels of both supportive and unsupportive ES; and finally, an Emotion Dismissing profile characterized by parents who exhibited the highest levels of unsupportive ES and the lowest levels of supportive ES. Only three other studies, to date, have utilized a person-centered approach to evaluating parental ES (Miller-Slough et al., 2015, 2017; O'Leary, 2020). As such, findings of the current study serve as an important contribution to a nascent area of the literature.

We also examined predictors of ES profiles related to family demographics and parent emotional competence. Mothers were less likely to be in the Emotion Dismissing profile compared to fathers, consistent with previous research showing that mothers tend to utilize more supportive ES strategies with youth relative to fathers (e.g., Baker et al., 2011; O'Leary et al., 2019). Although child sex did not predict class membership in our study, more research is 
needed to clarify how parent-child ES dynamics develop differently based on parents' sex, as well as when and how parent ES strategies vary by child sex (i.e., O’Leary et al., 2019). In addition, child age was explored as a potential predictor. Findings revealed that parents of younger children were more likely to be in the Emotion Coaching profile, while parents of older children were more likely to be in the Emotion Dismissing profile. This is in line with past work showing that "unsupportive" ES responses increase with child age (Klimes-Dougan \& Zeman, 2007; Labella, 2018). While subject to speculation of the utility of such practices at older ages and the surrounding context, it has been suggested that this may be the result of increases in parents' perceived danger of emotional expression as youth get older (Labella, 2018).

Interestingly, families of higher SES were less likely to be in the Emotion Dismissing profile but more likely to be in the Limited Engagement profile, potentially suggesting a "hands off' parenting approach around emotion. It is possible that our findings related to SES are tied to the resources these families have available to them. For example, high SES families may have more external supports from which they can draw upon for assistance, such as non-family caregivers (e.g., babysitters, extended family) or increased access to daycare or after-school programs for their children. Such external supports may decrease parents' caregiving demands and related parenting stress, lending to the lower likelihood of being in the Emotion Dismissing profile, but may also consequently decrease parents involvement as primary socializers in their children's lives (i.e., Luthar, 2001). Additional findings pertaining to family economics revealed that parents of families living within the federal poverty line had a higher likelihood of being in the Emotion Coaching profile. Although contrary to some literature (e.g., Shaffer et al., 2012), other studies indicate equivalent levels of emotional closeness across affluent and economically disadvantaged families (e.g., Luthar \& Latendresse, 2005). The final demographic predictor 
examined in the study was parent educational attainment, with data suggesting that higher attainment was associated with a lower likelihood of being in the Emotion Coaching profile.

Of particular importance were our findings related to parent emotional competence. Specifically, we found that parents who reported high levels of mindfulness had a significantly higher probability of being in the Emotion Coaching profile, which is consistent with findings from a prior longitudinal investigation conducted by McKee and colleagues (2018). As mindfulness is characterized by nonjudgmental awareness and acceptance of present experiences, it is plausible that a mindful disposition may be more broadly indicative of a parent's psychological resources, with mindful parents having a greater capacity to attend and be open to children's emotional experiences. We also found that the probability of being in the Emotion Dismissing profile substantially increased for parents with high levels of emotion dysregulation - a novel contribution to the literature. This, too, is logical, as dysregulated parents may be less likely to have the "emotional bandwidth" to engage in problem solving with the child, encourage the child to express emotion, or to have the resources to provide soothing. While perhaps not surprising, these findings underscore the importance of parent emotional competence and its influence on parent-child interactions (Hajal \& Paley, 2020) and child behavioral health, given the link between parenting stress and child behavior problems (Barroso et al., 2018). Current findings suggest that adaptive changes in ES within a family may be unlikely to occur unless efforts are made to support parents in strengthening psychological resources from which they can draw upon, particularly in difficult emotional interactions with their children. Fortunately, parent training programming with modules focused on parental wellbeing and/or emotion coaching is on the rise (see Salmon et al., 2014, for a review). 
Finally, we examined longitudinal relationships between profiles of parental ES strategies and child outcomes. Analyses revealed that the Emotion Dismissing profile was associated with the highest levels of child internalizing and externalizing problems and emotional negativity, while the Emotion Coaching profile was associated with the lowest levels of these outcomes. Although novel in the study's person-centered approach, these findings are largely in line with prior research demonstrating the effect of supportive and unsupportive ES on youth psychosocial adjustment (e.g., Katz et al., 2012; Miller-Slough \& Dunsmore, 2016). We also examined child age as a moderator of these relationships. In this respect, the associations between ES profile and child internalizing and externalizing problems were not moderated by child age, suggesting that the implications of ES remain salient throughout childhood. In contrast, the association between ES profile and child ER was moderated by child age, with the detrimental impact of the Emotion Dismissing profile being limited in children 3-5, moderate for children 6-8, and substantial for children 9-12 years-old. This is concerning given that the probability of being in the Emotion Dismissing profile increased with child age. Further, developmental shifts around the onset of puberty (i.e., 9-14) have been associated with increased vigilance towards socially affective information (e.g., unsupportive ES), resulting in states of emotional arousal and dysregulation in youth that likely contribute to trajectories of risk (Nelson et al., 2005). As such, our findings underscore the importance of identifying opportunities for early intervention, as the more exposure children have to maladaptive interactions with parents, the more likely they may be to exhibit emotional difficulties that could develop into entrenched psychopathology.

It is important to note that there remains much to learn about ES, particularly when it comes to qualifying strategies as "supportive" or "unsupportive", given previous research demonstrating less adaptive outcomes for traditionally-defined "supportive" strategies and more 
adaptive outcomes for so called "unsupportive" strategies in families of varying backgrounds (e.g., Castro et al., 2018; Lugo-Candelas et al., 2015). Previous research, for example, suggests that some racial and ethnic minority families, relative to European American families, may be more likely to use unsupportive responses to their children's negative emotions (e.g., Nelson et al., 2012), which notably, might be adaptive in the contexts of discrimination, racism, and prejudice (Dunbar et al., 2017), depending on other contextual risks (e.g., Zeman et al., 2016). Furthermore, O’Leary's (2020) person-centered approach suggests that racial/ethnic differences may play a role in the way that caregivers utilize combinations of socialization strategies. A major limitation of the current study is the restricted sample diversity, which precluded examining race/ethnicity as a demographic predictor of group membership or considering how it might interact with other demographic contexts. As such, more research is needed to answer questions about ES strategies and youth outcomes like "supportive for whom, and in what contexts?" as well as to explore the strength of associations and explanatory mechanisms.

Although our investigation expands our understanding of parental ES in important ways, data were from a single informant, increasing the risk of shared method bias. Despite this limitation, online recruitment through MTurk did enable the research team to obtain a greater representation of fathers in the sample - a demographic that has been historically more difficult to recruit in child psychopathology research (Parent et al., 2017) - and a large enough sample to examine effects of moderation by age. A second limitation of the study was the lack of diversity in the sample which prevented us from evaluating racial and ethnic differences across families. Additionally, only a small percentage of our sample met criteria for borderline or clinical psychopathology (Parent \& Forehand, 2017). Future work should enhance recruitment to allow for examinations of more representative samples as well as those at-risk of clinically significant 
symptomatology. The study benefited from its inclusion of multiple child age groups and personcentered approach - both of which allowed us to examine patterns in ES that would be important to our interpretation, especially for findings related to child outcomes. In addition, previous literature has largely failed to explore predictors of ES strategies between and within parents. Our exploration of family demographics and parent emotional competence therefore provides an important foundation on which future work can continue to expand, particularly given the highlighted variability in how some predictors relate to child outcomes. Lastly, it is important that future studies evaluate ES over longer spans of time, as our data points were only 4 months apart. Nonetheless, given that a majority of the research on ES is cross-sectional, we view the longitudinal design of our study as a strength.

Overall, our findings provide support for parental ES as an important process that promotes children's socioemotional functioning and also highlight the importance of parental emotional competence in the types of ES strategies utilized within families. Our person-centered approach suggests that meaningful change in a family exhibiting maladaptive patterns of ES is unlikely without first strengthening parents' emotional resources. Further, our identification of parent ES profiles and their respective associations with youth psychopathology provides a clearer picture of which families are more likely to be in need of intervention services, enhancing our ability to improve the trajectories of such families. However, more research is needed that provides an in-depth exploration of determinants of parental ES strategies to better identify targets for prevention and intervention. Only by doing so will we be able to distinguish how to best support parents in building an emotionally supportive family environment that fosters the adaptive development of youth. 


\section{References}

Achenbach, T.M., McConaughy, S.H., Ivanova, M.Y., Rescorla, L.A. (2011) Manual for the ASEBA Brief Problem Monitor тм (BPM). Burlington, VT: University of Vermont: University of Vermont Research Center for Children, Youth, and Families.

Baker, J. K., Fenning, R. M., \& Crnic, K. A. (2011). Emotion socialization by mothers and fathers: Coherence among behaviors and associations with parent attitudes and children's social competence. Social Development, 20(2), 412-430.

Bakk, Z., Tekle, F., \& Vermunt, J. (2013). Estimating the association between latent class membership and external variables using bias-adjusted three-step approaches. Sociological Methodology, 43, 272-311.

Barrera, M., Prelow, H. M., Dumka, L. E., Gonzales, N. A., Knight, G. P., Michaels, M. L., et al. (2002). Pathways from family economic conditions to adolescents' distress: Supportive parenting stressors outside the family, and deviant peers. Journal of Community Psychology, 30(2), 135-152.

Barroso, N. E., Mendez, L., Graziano, P. A., \& Bagner, D. M. (2018). Parenting stress through the lens of different clinical groups: A systematic review \& meta-analysis. Journal of Abnormal Child Psychology, 46(3), 449-461.

Breaux, R. P., Harvey, E. A., \& Lugo-Candelas, C. I. (2016). The role of parent psychopathology in emotion socialization. Journal of Abnormal Child Psychology, 44(4), 731-743.

Brown, K. W., \& Ryan, R. M. (2003). The benefits of being present: Mindfulness and its role in psychological well-being. Journal of Personality and Social Psychology, 84(4), 822-848.

Buhrmester, M.D., Kwang, T. \& Gosling, S.D. (2011). Amazon's Mechanical Turk: A new source of inexpensive, yet high-quality, data? Perspectives on Psychological Science, $6(1), 3-5$.

Cassano, M., Parrish, C., \& Zeman, J. (2007). Influence of gender on parental socialization of children's sadness regulation. Social Development, 16, 210 - 231. 
Castro, V. L., Halberstadt, A. G., \& Garrett-Peters, P. T. (2018). Changing tides: Mothers' supportive emotion socialization relates negatively to third-grade children's social adjustment in school. Social Development, 27(3), 510-525.

Chorpita, B. F., Reise, S., Weisz, J. R., Grubbs, K., Becker, K. D., \& Krull, J. L. (2010). Evaluation of the Brief Problem Checklist: Child and caregiver interviews to measure clinical progress. Journal of Consulting and Clinical Psychology, 78(4), 526-536.

Coatsworth, J. D., Duncan, L. G., Nix, R. L., Greenberg, M. T., Gayles, J. G., Bamberger, K. T., Berrena, E., \& Demi, M. A. (2015). Integrating mindfulness with parent training: effects of the Mindfulness-Enhanced Strengthening Families Program. Developmental Psychology, 51(1), 26-35.

Cummingham, J. N., Kliewer, W., \& Garner, P. W. (2009). Emotion socialization, child emotion understanding and regulation, and adjustment in urban African American families: Differential associations across child gender. Development and Psychopathology, 21, $261-283$.

Denham, S., \& Kochanoff, A. T. (2002). Parental contributions to preschoolers' understanding of emotion. Marriage \& Family Review, 34(3-4), 311- 343.

Dunbar, A. S., Leerkes, E. M., Coard, S. I., Supple, A. J., \& Calkins, S. (2017). An integrative conceptual model of parental racial/ethnic and emotion socialization and links to children's social-emotional development among African American families. Child Development Perspectives, 11, 16-22.

Eisenberg, N., Cumberland, A., \& Spinrad, T. L. (1998). Parental socialization of emotion. Psychological Inquiry, 9(4), 241-273.

Eisenberg, N., Spinrad, T. L., \& Eggum, N. D. (2010). Emotion-related self-regulation and its relation to children's maladjustment. Annual Review of Clinical Psychology, 6, 495-525

Fabes, R.A., Eisenberg, N., \& Bernzweig, J. (1990). The Coping with Children's Negative Emotions Scales. Arizona State University. 
Fainsilber Katz, L., Stettler, N., \& Gurtovenko, K. (2016). Traumatic stress symptoms in children exposed to intimate partner violence: The role of parent emotion socialization and children's emotion regulation abilities. Social Development, 25, 47-65.

Faro, A., McKee, L.G, Garcia, R.L., \& O'Leary, J.L. (2019) Emotion socialization, social connectedness and internalizing symptoms in emerging adults. Journal of Applied Developmental Psychology, 64, 101051.

Garside, R. B., \& Klimes-Dougan, B. (2002). Socialization of discrete negative emotions: Gender differences and links with psychological distress. Sex Roles: A Journal of Research, 47(3-4), 115-128.

Gottman, J.M., Katz, L.F., \& Hooven, C. (1996). Parental meta-emotion philosophy and the emotional life of families: Theoretical models and preliminary data. Journal of Family Psychology, 10, 243-268.

Hajal, N. J., \& Paley, B. (2020). Parental emotion and emotion regulation: A critical target of study for research and intervention to promote child emotion socialization. Developmental Psychology, 56(3), 403-417.

Halberstadt, A. G., Denham, S. A. and Dunsmore, J. C. (2001). Affective social competence. Social Development, 10, 79-119.

Hashima, P. Y., \& Amato, P. R. (1994). Poverty, social support, and parental behavior. Child Development, 65(2), 394-403.

Herbert, S., Harvey, E., Roberts, J., Wichowski, K., \& Lugo-Candelas, C. (2013). A randomized controlled trial of a parent training and emotion socialization program for families of hyperactive preschool-aged children. Behavior Therapy, 44, 302-16.

Hurrell, K. E., Houwing, F. L., \& Hudson, J. L. (2017). Parental metaemotion philosophy and emotion coaching in families of children and adolescents with an anxiety disorder. Journal of Abnormal Child Psychology, 45(3)569-582.

Katz, L.F., Maliken, A.C., \& Stettler, N.M. (2012) Parental meta-emotion philosophy: A review of research and theoretical framework. Child Development Perspectives, 6, 417-422. 
Klimes-Dougan, B., \& Zeman, J. (2007). Introduction to the special issue: Emotion socialization in childhood and adolescence. Social Development, 16, 203-209.

Labella, M.H. (2018). The sociocultural context of emotion socialization in African American families. Clinical Psychology Review, 59, 1-15.

Lo, Y., Mendell, N.R., \& Rubin, D.B. (2001) Testing the number of components in a normal mixture. Biometrika, 88(3), 767-778.

Luby, J. L., Barch, D. M., Whalen, D., Tillman, R., \& Freedland, K. E. (2018). A randomized controlled trial of parent-child psychotherapy targeting emotion development for early childhood depression. The American Journal of Psychiatry, 175(11), 1102-1110.

Lugo-Candelas, C. I., Harvey, E. A., \& Breaux, R. P. (2015). Emotion socialization practices in Latina and European-American mothers of preschoolers with behavior problems. Journal of Family Studies, 21, 144-162.

Lunkenheimer, Erika \& Shields, Ann \& Cortina, Kai. (2007). Parental emotion coaching and dismissing in family interaction. Social Development, 16, 232 - 248.

Luthar, S. S., \& Latendresse, S. J. (2005). Children of the affluent: Challenges to well-being. Current Directions in Psychological Science, 14(1), 49-53.

McElwain, N. L., Halberstadt, A. G., \& Volling, B. L. (2007). Mother- and father-reported reactions to children's negative emotions: Relations to young children's emotional understanding and friendship quality. Child Development, 78(5), 1407-1425.

McKee, L. G., Jones, D. J., Vaughn, A., \& Ward, D. (2015). Parental depressive symptoms and emotion regulation: Impact on emotion socialization. In L. G. McKee (Chair), Next steps in emotion socialization research: Exploring the context of parental stress and psychopathology. Symposium presented at the Society for Research in Child Development 2015 Biennial Meeting, Philadelphia, PA.

McKee, L. G., Parent, J., Zachary, C. R., \& Forehand, R. (2018). Mindful parenting and emotion socialization practices: Concurrent and longitudinal associations. Family Process, 57(3), $752-766$. 
Miller-Slough, R. \& Dunsmore, J. (2017). Parent and friend emotion socialization in adolescence: Associations with psychological adjustment. Adolescent Research Review, $1,1-19$.

Miller-Slough, R. L., Dunsmore, J. C., Zeman, J. L., Sanders, W. M., \& Poon, J. A. (2018). Maternal and paternal reactions to child sadness predict children's psychosocial outcomes: A family-centered approach. Social Development, 27, 495-509.

Morris, A. S., Silk, J. S., Steinberg, L., Myers, S. S., \& Robinson, L. R. (2007). The role of the family context in the development of emotion regulation. Social Development, 16(2), $361-388$.

Muthén, L. K., \& Muthén, B. O. (2017). Mplus: Statistical analysis with latent variables: User's guide (Version 8). Los Angeles, CA: Authors.

Nelson, E.E., Leibenluft, E., McClure, E., \& Pine, D.S. (2005). The social re-orientation of adolescence: A neuroscience perspective on the process and its relation to psychopathology. Psychological Medicine, 35, 163- 174.

Nelson, J. A., Leerkes, E. M., O’Brien, M., Calkins, S. D., \& Marcovitch, S. (2012). African American and European American mothers' beliefs about negative emotions and emotion socialization practices. Parenting: Science and Practice, 12, 22-41.

O'Neal, C. R., \& Magai, C. (2005). Do parents respond in different ways when children feel different emotions? The emotional context of parenting. Development and Psychopathology, 17(2), 467-487.

O’Leary, J. L. (2020). The role of emotion socialization and emotion regulation in protecting community violence-exposed urban youth. [Doctoral dissertation, Clark University]. Available from ProQuest Dissertations and Theses database.

O’Leary, J. L., McKee, L. G., \& Faro, A. L. (2019). Guilt and shame: Explaining associations between emotion socialization and emerging adult well-being. Family Relations, 68, $608-623$. 
Parent, J., \& Forehand, R. (2017). The Multidimensional Assessment of Parenting Scale (MAPS): Development and psychometric properties. Journal of Child and Family Studies, 26(8), 2136-2151.

Parent, J., Forehand, R., Pomerantz, H., Peisch, V., \& Seehuus, M. (2017). Father participation in child psychopathology research. Journal of Abnormal Child Psychology, 45(7), 12591270.

Salmon, K., Dittman, C., Sanders, M., Burson, R., \& Hammington, J. (2014). Does adding an emotion component enhance the Triple P-Positive Parenting Program? Journal of Family Psychology, 28(2), 244-252.

Shaffer, A., Suveg, C., Thomassin, K., \& Bradbury, L. L. (2012). Emotion socialization in the context of family risks: Links to child emotion regulation. Journal of Child and Family Studies, 21(6), 917-924.

Shields, A., \& Cicchetti, D. (1997). Emotion regulation among school-age children: The development and validation of a new criterion Q-sort scale. Developmental Psychology, 33(6), 906-916.

Vermunt, J.K. (2010). Latent class modeling with covariates: Two improved three-step approaches. Political Analysis, 18, 450-469.

Victor, S. E., \& Klonsky, E. D. (2016). Validation of a Brief Version of the Difficulties in Emotion Regulation Scale (DERS-18) in five samples. Journal of Psychopathology and Behavioral Assessment, 38, 582-589.

Zeman, J., Dallaire, D., \& Borowski, S. (2016). Socialization in the context of risk and psychopathology: Maternal emotion socialization in children of incarcerated mothers. Social Development, 25(1), 66-81. 
Table 1.

LPA Model Fit Indices.

\begin{tabular}{cccc|cc}
\hline & & \multicolumn{2}{c|}{ Parsimony Criteria } & \multicolumn{2}{c}{ LRT p Value } \\
\cline { 2 - 6 } Profiles Entropy & AIC & BIC & LMRa & BLRT \\
\hline 1 & -- & 5399 & 5445 & -- & -- \\
2 & .86 & 4730 & 4826 & .000 & .000 \\
3 & .85 & 4520 & 4666 & .002 & .000 \\
4 & .83 & 4418 & 4613 & .347 & .000 \\
5 & .86 & 4340 & 4594 & .020 & .000 \\
6 & .88 & 4311 & 4605 & .072 & .000 \\
7 & .87 & 4280 & 4624 & .558 & .040 \\
8 & .88 & 4254 & 4648 & .232 & .667 \\
\hline
\end{tabular}


Table 2.

Predictors of profile membership.

\begin{tabular}{lcccc}
\hline Effect & Estimate & SE & OR & $\boldsymbol{p}$ Value \\
\hline Limited vs. Emotion Dismissing & & & & \\
Parent Sex & -2.17 & .696 & .114 & .002 \\
Child Age & -.234 & .112 & .791 & .036 \\
Parent Emotion Dysregulation & -.731 & .182 & .481 & .000 \\
Parent Mindfulness & .044 & .035 & 1.04 & .211 \\
Parent Education & 2.07 & .794 & 7.88 & .009 \\
Family Poverty Status & -2.58 & .742 & .076 & .001 \\
Moderate vs. Emotion Dismissing & & & & \\
Parent Sex & -2.28 & .732 & .102 & .002 \\
Child Age & -.119 & .098 & .887 & .222 \\
Parent Emotion Dysregulation & -.612 & .162 & .542 & .000 \\
Parent Mindfulness & -.018 & .029 & .982 & .541 \\
Parent Education & 1.35 & .661 & 3.85 & .042 \\
Family Poverty Status & -1.92 & .690 & .147 & .005 \\
Emotion Coaching vs. Emotion Dismissing & & & & \\
Parent Sex & -2.69 & .726 & .068 & .000 \\
Child Age & -.301 & .106 & .740 & .004 \\
Parent Emotion Dysregulation & -.744 & .198 & .475 & .000 \\
Parent Mindfulness & .107 & .033 & 1.11 & .001 \\
Parent Education & 2.23 & .793 & 9.33 & .005 \\
Family Poverty Status & -1.77 & .778 & .170 & .023 \\
\hline
\end{tabular}




\section{Table 3.}

Distal child psychopathology outcomes

\begin{tabular}{lccc}
\hline Effect & Wald $\boldsymbol{\chi} \mathbf{d}$ & $\mathbf{d f}$ & $\boldsymbol{p}$ Value \\
\hline Cross-sectional & & & \\
$\quad$ Emotional negativity & 33.86 & 3 & .000 \\
Emotion regulation & 65.53 & 3 & .000 \\
Internalizing & 25.36 & 3 & .000 \\
Externalizing & 34.54 & 3 & .000 \\
Longitudinal & & & \\
$\quad$ Emotional negativity & 12.27 & 3 & .007 \\
Emotion regulation & 4.74 & 3 & .192 \\
Internalizing & 31.44 & 3 & .000 \\
$\quad$ Externalizing & 18.29 & 3 & .001 \\
Stability & & & \\
$\quad$ Emotional negativity & 6.86 & 3 & .077 \\
Emotion regulation & .571 & 3 & .903 \\
Internalizing & 74.76 & 3 & .000 \\
Externalizing & 27.18 & 3 & .000 \\
\hline
\end{tabular}

Note. Cross-sectional refers to the mean differences at wave three (8-month). Longitudinal refers to mean differences at wave four (12-month). Stability refers to differences in the association between waves three and four across groups. 
Table 4.

Detailed results for distal child outcomes.

\begin{tabular}{|c|c|c|c|c|c|c|c|c|c|c|c|c|}
\hline \multirow[b]{2}{*}{ Effect } & \multicolumn{3}{|c|}{ Limited } & \multicolumn{3}{|c|}{ Moderate } & \multicolumn{3}{|c|}{ Emotion Coaching } & \multicolumn{3}{|c|}{ Emotion Dismissing } \\
\hline & Estimate & SE & $p$ & Estimate & SE & $p$ & Estimate & SE & $p$ & Estimate & SE & $p$ \\
\hline INT_8 $\rightarrow$ INT_12 & .222 & .036 & .000 & .670 & .096 & .000 & -.016 & .044 & .711 & .977 & .184 & .000 \\
\hline EXT_8 $\rightarrow$ EXT_12 & .151 & .096 & .117 & .724 & .094 & .000 & .444 & .045 & .000 & .916 & .229 & .000 \\
\hline Mean INT_8 & .603 & .207 & .003 & 1.81 & .235 & .000 & .623 & .163 & .000 & 1.93 & .427 & .000 \\
\hline Mean EXT_8 & 1.31 & .269 & .000 & 2.49 & .286 & .000 & .658 & .219 & .003 & 3.03 & .599 & .000 \\
\hline Intercept INT_12 & .121 & .090 & .180 & 1.34 & .304 & .000 & .236 & .093 & .011 & .806 & .468 & .085 \\
\hline Intercept EXT_12 & .752 & .197 & .000 & 1.07 & .393 & .007 & .040 & .110 & .690 & 1.14 & 1.11 & .305 \\
\hline Resid. Variance INT_12 & .143 & .057 & .012 & 1.95 & .406 & .000 & .175 & .048 & .000 & 2.98 & 1.21 & .014 \\
\hline Resid. Variance EXT_12 & .908 & .303 & .003 & 3.24 & 1.19 & .007 & .143 & .103 & .165 & 8.44 & 3.46 & .015 \\
\hline NEG_8 $\rightarrow$ NEG_12 & .917 & .076 & .000 & .694 & .129 & .000 & .661 & .070 & .000 & .899 & .161 & .000 \\
\hline ER_8 $\rightarrow$ ER_12 & .747 & .116 & .000 & .669 & .166 & .000 & .678 & .230 & .003 & .592 & .171 & .001 \\
\hline Mean NEG_8 & 24.45 & .914 & .000 & 27.18 & .845 & .000 & 22.01 & .831 & .000 & 29.48 & 1.07 & .000 \\
\hline Mean ER_8 & 26.04 & .503 & .000 & 26.15 & .439 & .000 & 29.04 & .437 & .000 & 22.51 & .700 & .000 \\
\hline Intercept NEG_12 & 1.96 & 1.57 & .211 & 7.43 & 3.28 & .000 & 6.80 & 1.64 & .000 & 4.41 & 4.90 & .369 \\
\hline Intercept ER_12 & 6.92 & 3.13 & .027 & 8.72 & 4.48 & .000 & 9.70 & 6.92 & .161 & 9.57 & 4.17 & .022 \\
\hline Resid. Variance NEG_12 & 4.83 & 1.49 & .001 & 16.52 & 2.98 & .000 & 9.62 & 1.80 & .000 & 22.99 & 7.09 & .001 \\
\hline Resid. Variance ER_12 & 5.06 & 1.40 & .000 & 6.31 & 1.14 & .000 & 3.65 & .810 & .000 & 12.19 & 3.90 & .002 \\
\hline
\end{tabular}

Note. INT $=$ internalizing, EXT $=$ externalizing, $\mathrm{NEG}=$ emotional negativity, $\mathrm{ER}=$ emotion regulation $\rightarrow$ regression, Resid. $=$ Residual. 
Table 5.

Interaction between child age and emotion socialization profile

\begin{tabular}{|c|c|c|c|c|c|c|}
\hline \multirow[b]{2}{*}{ DV: 12-Month Emotion Negativity } & \multirow[b]{2}{*}{ Estimate } & \multirow[b]{2}{*}{ SE } & \multirow[b]{2}{*}{$p$} & \multirow[b]{2}{*}{ Stand. Estimate } & \multicolumn{2}{|c|}{$95 \% \mathrm{CI}$} \\
\hline & & & & & Lower & Upper \\
\hline Intercept ${ }^{a}$ & 1.922 & 2.742 & 0.484 & & & \\
\hline \multicolumn{7}{|l|}{ Profile: } \\
\hline Limited - Emotion Dismissing & 4.353 & 2.696 & 0.108 & -0.268 & -0.551 & 0.015 \\
\hline Moderate - Emotion Dismissing & 4.911 & 2.729 & 0.074 & -0.335 & -0.607 & -0.064 \\
\hline $\begin{array}{l}\text { Emotion Coaching - Emotion } \\
\text { Dismissing }\end{array}$ & 3.489 & 2.656 & 0.191 & -0.470 & -0.759 & -0.180 \\
\hline 8-Month Emotional Negativity & 0.773 & 0.047 & $<.001$ & 0.733 & 0.644 & 0.821 \\
\hline Child Age & 0.777 & 0.282 & 0.006 & 0.339 & 0.096 & 0.582 \\
\hline \multicolumn{7}{|l|}{ Child Age 怾 Profile: } \\
\hline $\begin{array}{l}\text { Child Age } * \text { (Limited - Emotion } \\
\text { Dismissing) }\end{array}$ & -0.869 & 0.331 & 0.009 & -0.379 & -0.663 & -0.095 \\
\hline $\begin{array}{l}\text { Child Age } * \text { (Moderate }- \text { Emotion } \\
\text { Dismissing) }\end{array}$ & -1.011 & 0.333 & 0.003 & -0.441 & -0.727 & -0.155 \\
\hline $\begin{array}{l}\text { Child Age } * \text { (Emotion Coaching - } \\
\text { Emotion Dismissing) }\end{array}$ & -0.934 & 0.327 & 0.005 & -0.408 & -0.689 & -0.126 \\
\hline
\end{tabular}

Note. $\mathrm{a}=$ Emotion Dismissing reference 


\section{Figure 1.}

Latent profile subscale means.

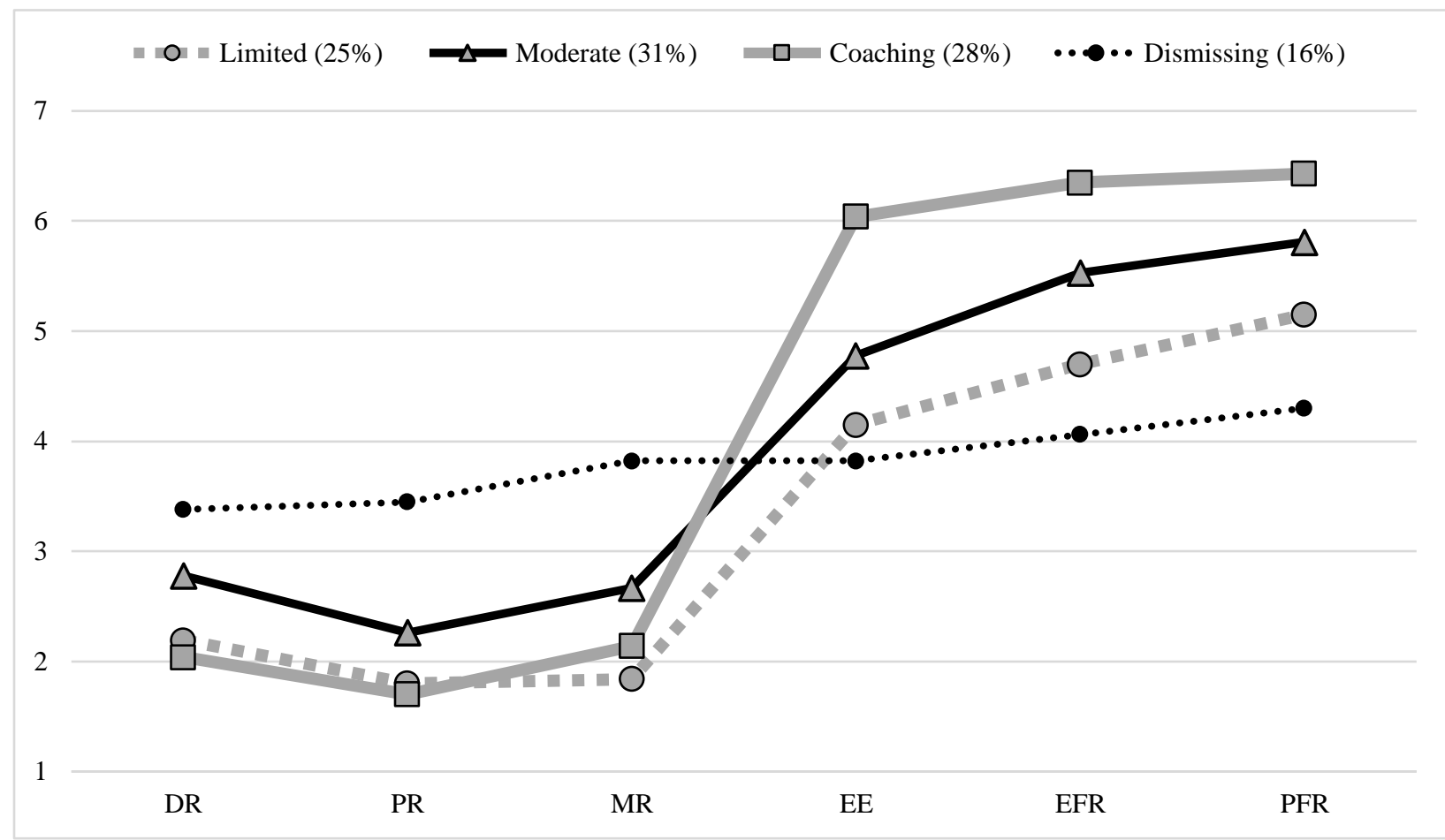

Note $. \mathrm{DR}=$ distress reactions, $\mathrm{PR}=$ punitive reactions, $\mathrm{MR}=$ minimization reactions, $\mathrm{EE}=$ expressive encouragement, EFR = emotion-focused reactions, and PFR = problem-focused reactions. The $\mathrm{Y}$ axis is the Likert scale of the emotion socialization scale. 


\section{Figure 2.}

Latent profile predicators: Parent mindfulness and emotional dysregulation.

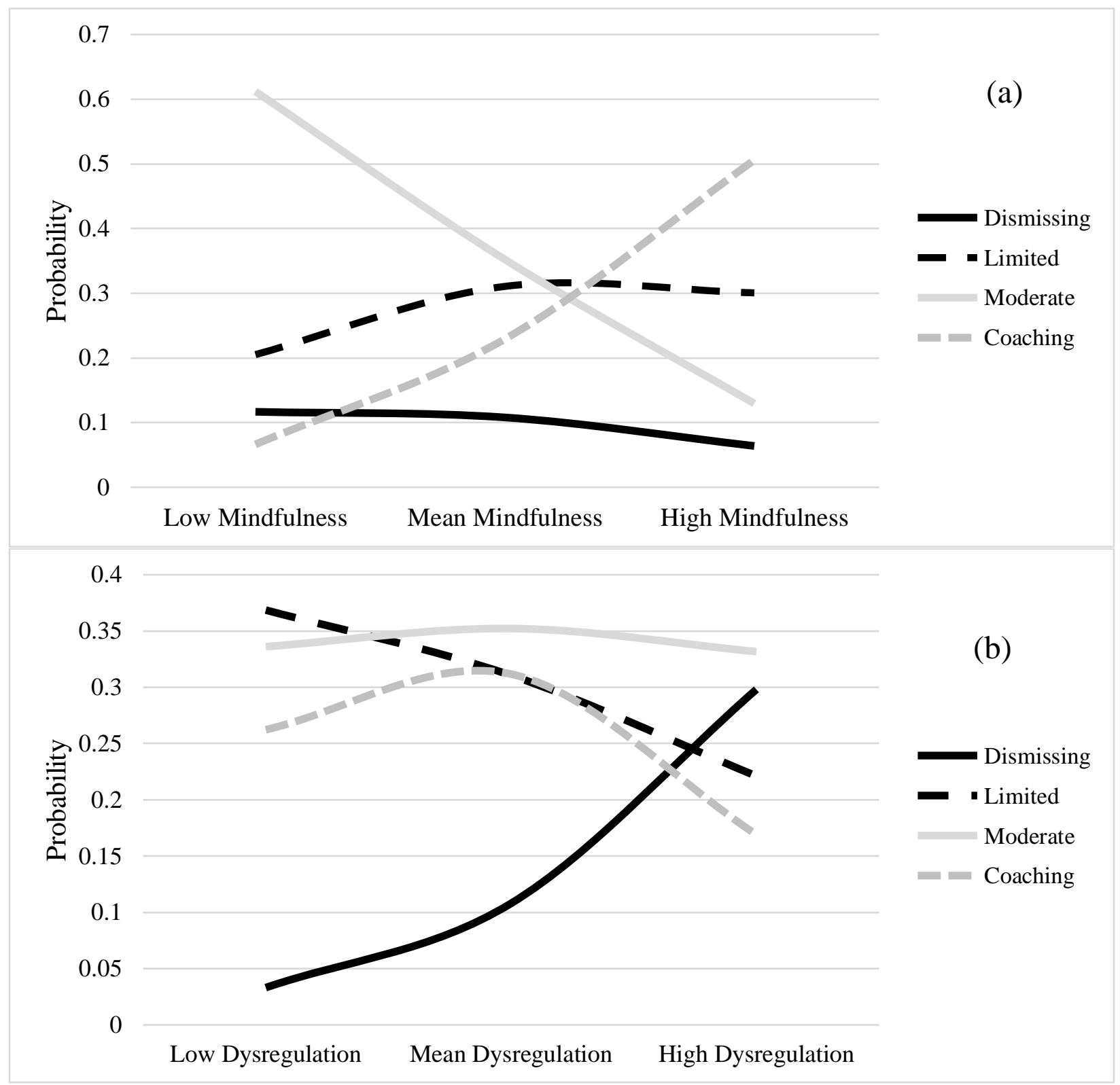


EMOTION SOCIALIZATION \& YOUTH PSYCHOPATHOLOGY

Figure 3.

Mean Z-scores on child outcomes for each ES profile.

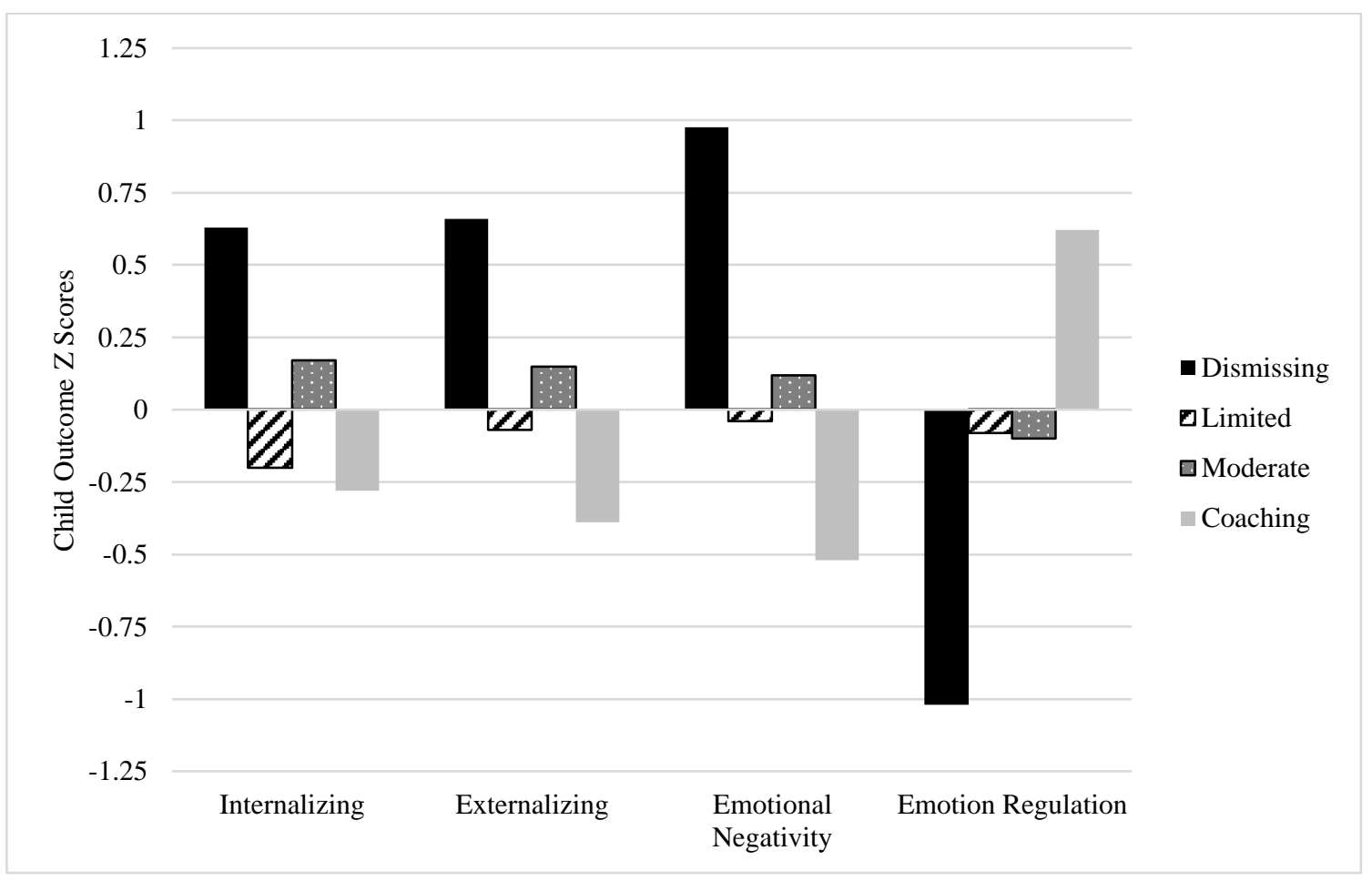

